\title{
THE EXISTENCE AND UNIQUENESS OF NONSTATIONARY IDEAL INCOMPRESSIBLE FLOW IN BOUNDED DOMAINS IN $R_{3}$
}

BY

\author{
H. S. G. SWANN
}

\begin{abstract}
It is shown here that the mixed initial-boundary value problem for the Euler equations for ideal flow in bounded domains of $R_{3}$ has a unique solution for a small time interval. The existence of a solution is shown by converting the equations to an equivalent system involving the vorticity and applying Schauder's fixed point theorem to an appropriate mapping.
\end{abstract}

Introduction. The purpose of this paper is to prove the existence and uniqueness of classical solutions to the mixed initial-boundary value problem for the Euler equations for an ideal incompressible fluid in bounded domains of $R_{3}$ for a small time interval. This has been shown by Ebin and Marsden [3] for compact Riemannian manifolds possibly with boundary. In this paper we obtain a new proof by converting the equations to an equivalent system involving the vorticity and solving this by using the Schauder fixed point theorem. The technique is similar to that of Kato [4] where he proved existence of ideal flow in bounded domains of the plane for an arbitrary time interval. The author also used a similar method to obtain an existence result for ideal flow in all $R_{3}$ by showing that a solution is the limit of Navier-Stokes flow as the viscosity goes to zero [7]. Existence of a solution for a short time in all $R_{3}$ was originally shown by Lichtenstein [5].

The Euler equations for ideal incompressible flow in a domain $D$ in $R_{3}$ with boundary $b D$ are

$$
\partial v / \partial t+(v \cdot \operatorname{grad}) v=-\operatorname{grad} P+B, \quad \nabla \cdot v=0,
$$

with constraints $v \cdot n=0$ on $b D$ and $v(0)=A$, where $x$ is a point in $D \cup b D$; $t \in[0, T], v(x, t)$ is the velocity vector, $P(x, t)$ is the (scalar) pressure, $B(x, t)$ is the external force field vector, $n(x)$ is the outward normal vector to $b D$ and $A(x)$ is the initial velocity vector.

By formally computing the curl of $\left(E^{\prime}\right)$ we get the system

Presented to the Society, January 18, 1972 under the title The existence and uniqeness of nonstationary ideal flow in bounded domains in $R_{3}$; received by the editors October 12, 1971 and, in revised form, March 20, 1972.

AMS (MOS) subject classifications (1970). Primary 35F25, 35F30, 35Q05, 76C05.

Key words and phrases. Nonstationary ideal flow, Euler equations. 
(E)

(a) $\quad \partial w / \partial t+(v \cdot \operatorname{grad}) w-(w \cdot \operatorname{grad}) v=\nabla \times B \equiv b$,

(b) $\quad w(x, 0)=\nabla \times A(x) \equiv a(x)$,

(c) $\nabla \times v=w, \quad \nabla \cdot v=0$,

(d) $\quad v \cdot n=0 \quad$ on $b D$.

We solve this system to obtain a solution to $\left(E^{\prime}\right)$ in three steps:

In $\S I$ we show that if $w$ is in an appropriate class of functions, then there is a function $v \equiv F_{1}(w)$ that solves (E), (c) and (d).

In $\S$ II we show that for $v=F_{1}(w)$ there is a solution, denoted $F_{2}(v)$, to (E), (a) and (b).

In §III we show that the mapping $F_{2}\left(F_{1}(\cdot)\right)$ maps a closed convex compact set of functions in a Banach space continuously into itself provided we restrict the time interval of solution $[0, T]$. Hence we can apply Schauder's fixed point theorem to obtain a function $w$ such that $w=F_{2}\left(F_{1}(w)\right)$ and from this a solution to $(\mathrm{E})$. Finally we show that this gives a classical solution to $\left(\mathrm{E}^{\prime}\right)$ with $v$ unique and $P$ unique up to an arbitrary function of $t$ which may be added to $P$.

The author is indebted to Professor Tosio Kato for his many helpful suggestions and comments.

The following notation is used in this paper:

$D$ is the domain, connected, open and bounded in $R_{3}$ with closure $\bar{D}$ and boundary $b D$ of class $C^{3+q}$ for some $q, 0<q<1$ (see [6, p. 300]). $\bar{D} \times[0, T]$ is denoted $(\bar{D} ; T)$, where $T>0$ is arbitrary.

$C^{q, r}(\bar{D} ; T)$ is the Banach space of vector-valued functions from $(\bar{D} ; T)$ to $R_{\mathbf{3}}$ with continuous space derivatives of order $\leq[q]$ (denoted conventionally $D_{x}^{\alpha}$ ) continuously extendable to $\bar{D}$ and continuous time derivatives of order $\leq[r]$, where $[q]$ and $[r]$ are the largest integers less than or equal to $q$ and $r$ respectively. Space derivatives of order $[q]$ are Hölder-continuous with exponent $q-[q]$ and time derivatives of order $[r]$ are Hölder-continuous with exponent $r-[r]$.

If $|v|^{2}=\sum_{i=1}^{3}\left|v_{i}\right|^{2}$ and

$$
K^{q^{\prime}, r^{\prime}}(v)=\sup _{\substack{t, t^{\prime} \in[0, T] ; \neq \neq t^{\prime} \\ x, x^{\prime} \in \bar{D} ; x \neq x^{\prime} \\\left|x-x^{\prime}\right|<1, t, t-t^{\prime} \mid<1}} \frac{\left|v\left(x^{\prime}, t^{\prime}\right)-v(x, t)\right|}{\left|x-x^{\prime}\right|^{q^{\prime}}+\left|t-t^{\prime}\right|^{\prime}}
$$

we define a norm for $C^{q, r}(\bar{D} ; T)$ as

$$
\begin{aligned}
\|v\|_{C^{\alpha, r}}^{2}= & \sup _{\substack{x \in \bar{D} \\
t \in[0, T]}} \sum_{\substack{\alpha \mid \leq[q] \\
\beta \leq[r]}}\left|D_{x}^{\alpha}\left(\frac{d}{d t}\right)^{\beta} v(x, t)\right|^{2} \\
& +\sum_{\substack{|\alpha|=[q] \\
\beta=[r]}}\left[K^{q-[q], r-[r]}\left(D_{x}^{\alpha}\left(\frac{d}{d t}\right)^{\beta} v\right)\right]^{2} .
\end{aligned}
$$


When we allow $t \in[0, T], t>0$, to vary we will note this by using the norm notation \|\|$_{C^{q,}(\bar{D} ; t)}$ defined in the obvious way. For convenience, \|\|$_{C}=\|\|_{C^{0,0}}$. We occasionally use analogously defined $C^{q}(\bar{D})$ with only space variables $(t \in[0, T]$ is fixed if present $)$.

$v_{, x}$ is the array with $i$ th row $\left(\partial v_{i} / \partial x_{1}, \partial v_{i} / \partial x_{2}, \partial v_{i} / \partial x_{3}\right), i=1,2,3$.

$$
\left\|v_{, x}\right\|_{(\cdot)}^{2}=\sum_{i, j=1,3}\left|\frac{\partial v_{i}}{\partial x_{j}}\right|_{(\cdot)}^{2}
$$

for various norms $(\cdot)$.

$L_{2}(D)$ is the Hilbert space of vector-valued functions $u$ over $D$ with innerproduct denoted

$$
\left(u^{1}, u^{2}\right)=\sum_{i=1,3} \int_{D} u_{i}^{1} u_{i}^{2} d V
$$

$C_{n}^{q}=\left\{f \in C^{q}(\bar{D}) \mid f(x)=g(x) n\right.$ for some scalar-valued function $g(x)$ on $\left.b D\right\}$

$$
C_{\tau}^{q}=\left\{f \in C^{q}(\bar{D}) \mid f \cdot n=0 \text { on } b D\right\}
$$

$H_{n}$ is the finite dimensional subspace of $C_{n}^{1+q}$ of functions $h$ such that $\nabla \times h=0$ and $\nabla \cdot h=0(0<q<1)$.

$H_{\tau}$ is the finite dimensional subspace of $C_{\tau}^{1+q}$ of functions $h$ such that $\nabla \times h=0$ and $\nabla \cdot h=0$. We let $\left\{h^{i}\right\}, i=1, \ldots, m$, be an $L_{2}$-orthonormal basis of $H_{\tau}$. (See [6, Chapter 7] for a further discussion of these subspaces.)

I.

Theorem I-1. Let $A \in C^{1+q}(\bar{D})$ and $B \in C^{1+q, 0}(\bar{D} ; T)$. If $w \in C^{q, q}(\bar{D} ; T)$ and $0=\nabla \cdot w$ (generalized) and, for fixed $t, w \in L_{2}(D) \ominus H_{n}$, then there exists a unique $v \in C^{1+q, 0}(\bar{D} ; T)$ such that

$$
\nabla \cdot v=0, \quad \nabla \times v=w, \quad v \cdot n=0 \quad \text { on } b D
$$

and

$$
(d / d t)(v, H)=(-(v \cdot \operatorname{grad}) v+B, H), \quad(v(0), H)=(A, H)
$$

for any $H \in H_{\tau}$.

If we let $v=u+h$ where $u \in L_{2}(D) \ominus H_{\tau}$ and $h \in H_{\tau}$ then there are constants $C_{1}(r), C_{3}(r)$ and $C_{4}(r)$ depending on $D$ and $r, 0<r \leq q$, and a constant $C_{2}$ depending on $D$ such that 


$$
\|u\|_{C^{1+r, 0}} \leq C_{1}(r)\|w\|_{C^{r, 0}}
$$

and

$$
\|h\|_{C^{1+, 0}} \leq\left(C_{2}\left(\left\|A_{H}\right\|_{C}+T\left\|B_{H}\right\|_{C}\right)+C_{3}(r)\|w\|_{C^{r, 0}}\right) \exp \left(C_{4}(r)\|w\|_{C^{r, 0}} T\right)
$$

where $A_{H}$ and $B_{H}$ are the projections of $A$ and $B$ respectively onto $H_{\tau}$.

We denote this unique $v=u+h=F_{1}(w)$.

Proof. We will have recourse to the following representations of $f \in C^{r}(\bar{D})$ at several points in this paper.

When interpreted as a statement about vector-valued functions Theorem 7.7.4 of Morrey [6] gives the existence of vector-valued functions $p_{f}$ and $\overline{p_{f}^{-}}$for any $f \in C^{r}(\bar{D})$ such that

$$
\begin{aligned}
& f=\nabla \times\left(\nabla \times p_{f}\right)-\nabla\left(\nabla \cdot p_{f}\right)+h_{f}, \\
& f=\nabla \times\left(\nabla \times \overline{p_{f}^{-}}\right)-\nabla\left(\nabla \cdot \overline{p_{f}^{-}}\right)+\overline{h_{f}^{-}}
\end{aligned}
$$

with $h_{f}^{-} \in H_{\tau}, h_{f} \in H_{n}, \nabla \times p_{f}^{-}$and $p_{f} \in C_{n}^{1+r} ; \nabla \times p_{f}$ and $p_{f}^{-} \in C_{\tau}^{1+r} ; \nabla \cdot p_{f}$ and $\nabla \cdot p_{f}^{-} \in C^{1+r}(\bar{D})$ (as scalar-valued functions) and $\nabla \cdot p_{f}=0$ on $b D$. The three terms of each representation are mutually $L_{2}$-orthogonal.

Since $w \in C^{q, q}(\bar{D} ; T)$ and $w \in L_{2}(D) \ominus H_{n}, w=\nabla \times\left(\nabla \times p_{w}\right)-\nabla\left(\nabla \cdot p_{w}\right)$ with these two terms orthogonal. However, since $(w, \nabla q)=0$ for all smooth scalar-valued functions $q$ with compact support in $D$, a conventional mollifier and limit argument can be used to establish that $\left(w, \nabla\left(\nabla \cdot p_{w}\right)\right)=0$, for $\nabla \cdot p_{w}$ is zero on $b D$. Hence $\nabla\left(\nabla \cdot p_{w}\right)=0$ and $w=\nabla \times\left(\nabla \times p_{w}\right)$. Letting $u=\nabla \times p_{w}$ and interpreting it as a two-form, Lemma 7.5.3 of [6] can be used to show that $u \in L_{2}(D) \ominus H_{r}$, which easily establishes uniqueness as well.

The operator taking $w$ into $u$ (for fixed $t$ ) is clearly a closed linear operator from the subspace $Q$ of $C^{r}(\bar{D}), 0<r \leq q$, defined by

$$
Q=\left\{w \in C^{r}(\bar{D}) \cap\left(L_{2}(\bar{D}) \ominus H_{n}\right) \mid 0=\nabla \cdot w \text { generalized }\right\}
$$

into $C^{1+r}(\bar{D})$ with associated norms. Hence, by the closed graph theorem, a constant $C_{1}(r)$ exists satisfying (i) for fixed $t \in[0, T]$. However, the linearity of the operator easily establishes that $u \in C^{1+r, 0}(\bar{D} ; T)$ and (i) follows.

With $u$ defined from $w$ by the preceding, we let $v=u+\sum_{i=1}^{m} g^{i}(t) h^{i}$ where the scalars $g^{i}(t)$ are unknown and $\left\{h^{i}\right\}$ is a basis of $H_{\tau}$. Since $\left(\nu, h^{i}\right)=g^{i}(t)$, we can form the ordinary differential equations in $\left\{g^{i}\right\}$ :

$$
\begin{aligned}
d g^{i} / d t & =(d / d t)\left(v, h^{i}\right)=\left(-(v \cdot \operatorname{grad}) v+B, h^{i}\right) \\
g^{i}(0) & =\left(v(0), h^{i}\right)=\left(A, h^{i}\right), \quad i=1, \ldots, m .
\end{aligned}
$$


An argument similar to that in [4, p. 195] gives, for suitable constants $K_{1}$ and $K_{2}$ which depend on $D$,

$$
\frac{1}{2} \frac{d}{d t} \sum g^{i}(t)^{2} \leq K_{1}\left(\left\|B_{H}\right\|_{C}+\|u\|_{C}^{2}\right)\left(\sum g^{i}(t)^{2}\right)^{1 / 2}+K_{2}\|u\|_{C} \sum g^{i}(t)^{2} .
$$

This leads to the estimate

$$
\begin{aligned}
\left(\sum g^{i}(t)^{2}\right)^{1 / 2} \leq & \left(\left\|A_{H}\right\|_{L_{2}}+t K_{1}\left\|B_{H}\right\|_{C}\right) \exp \left(t K_{2}\|u\|_{C}\right) \\
& +\frac{K_{1}}{K_{2}}\|u\|_{C}\left(\exp \left(t K_{2}\|u\|_{C}\right)-1\right) .
\end{aligned}
$$

Thus $h(t)=\sum g^{i}(t) h^{i}$ exists in $[0, T]$. This inequality can be combined with the estimate (i) to yield (ii). Thus $v \equiv u+h \equiv F_{1}(w)$ exists and is unique and has the required properties.

II. We wish to establish

Theorem II-1. If $v \in C^{1+q, 0}(\bar{D} ; T), \nabla \cdot v=0, v \cdot n=0$ on $b D, a \in C^{q}(\bar{D})$, $b \in C^{q, 0}(\bar{D} ; T)$, then we can construct $w \in C^{q, q}(\bar{D} ; T)$ uniquely with $w(x, 0)=a(x)$ and, for all $f \in C^{\mathrm{l}}(\bar{D})$,

$$
(d / d t)(w, f)=(w,(v \cdot \operatorname{grad}) f)+((w \cdot \operatorname{grad}) v, f)+(b, f) .
$$

The following theorem from [1, p. 105] is sufficient to establish our estimates:

Theorem A. Let $E$ be some bounded domain in $R_{3}$. If $Y(x, t) \in C^{1,0}(\bar{E}, T)$ and $Z(x, t) \in C(\bar{E}, T)$ and

$$
y(t) \text { solves } d y / d t=Y(y, t)
$$

and

$$
z(t) \text { solves } d z / d t=Z(z, t)
$$

then

$$
|y(t)-z(t)| \leq\left\{|y(s)-z(s)|+|t-s|\|Y-Z\|_{C}\right\} \exp \left(T\left\|Y_{, x}\right\|_{C}\right)
$$

We begin by solving for the streamlines of the "flow" $v$.

Lemma II-1. If $v \in C^{1+q, 0}(\bar{D} ; T), \nabla \cdot v=0, v \cdot n=0$ on $b D$ and $X(x, s ; t)$ solves $d X / d t=v(X, t)$ with initial condition $X(x, s ; s)=x_{2}$ then (i) $X(x, s ; t)$ is unique and continuously differentiable in $x \in D$ and $s, t \in[0, T]$ and 
(a)

$$
\left|X(x, s ; t)-X\left(x^{\prime}, s ; t\right)\right| \leq\left|x-x^{\prime}\right| \exp \left(T\left\|v_{, x}\right\|_{C}\right)
$$

$$
\left|X(x, s ; t)-X\left(x, s^{\prime} ; t^{\prime}\right)\right| \leq\left(\left|t-t^{\prime}\right|+\left|s-s^{\prime}\right|\right)\|v\|_{C} \exp \left(T\left\|v_{, x}\right\|_{C}\right)
$$

and (ii) for fixed $t$ and $s, X(\cdot, s ; t): x \rightarrow X(x, s ; t)$ is a one-to-one measurepreserving map of $\bar{D}$ onto $\bar{D}$ such that $\operatorname{det}\left(X_{, x}\right)=1$ and $b D \rightarrow b D$;

$$
X(\cdot, s ; s)=\text { Identity, } \quad X(\cdot, s ; t)=[X(\cdot, t ; s)]^{-1} .
$$

Proof. Since $v \in C^{1+q, 0}(\bar{D} ; T)$, there exists a unique local solution for the streamlines; they can be continued until they reach $b D$. Hence to show that $\bar{D}$ is mapped into $\bar{D}$ and $b D$ into $b D$ it suffices to show that a solution starting on $b D$ remains there, i.e. on the "sidewall" $b D \times[0, T]$. Since $v \cdot n=0$ on $b D$ and $b D$ is smooth we can define a system of ordinary differential equations on $b D \times[0, T]$ whose solutions are solutions of the original system and will stay on the "sidewall" by definition and this is sufficient to obtain our result.

The remainder of (ii) and the differentiability stated in (i) are standard results of ordinary differential equations since $v \in C^{1+q, 0}(\bar{D}, T)$ and $\nabla \cdot v=0$. (See $[2$, Chapter 1, §7].)

The estimates in (i) are standard or follow from Theorem A; for example

$$
\begin{aligned}
\| X(x, s ; t)-X\left(x, s^{\prime} ; t\right) \mid & =\left|X(x, s ; t)-X\left(X\left(x, s^{\prime} ; s\right), s ; t\right)\right| \\
& \leq\left|X\left(x, s^{\prime} ; s^{\prime}\right)-X\left(x, s^{\prime} ; s\right)\right| \exp \left(T\left\|v_{, x}\right\|_{C}\right) \\
& \leq\left|s-s^{\prime}\right|\|v\|_{C} \exp \left(T\left\|v_{, x}\right\|_{C}\right) .
\end{aligned}
$$

To obtain a solution to the equations of Theorem II-1 we first solve the system (O) of ordinary differential equations:

$$
d y / d t-v_{, x}(X(x, s ; t), t) y=b(X(x, s ; t), t)
$$

with initial condition $y(x, s ; 0)=a(X(x, s ; 0))$. We will then establish that $w(x, t)$ $\equiv y(x, t ; t)$ is the solution required by Theorem II- 1 .

Lemma II-2. Under the conditions assumed for $a, b$ and $v$ in Theorem II-1, there is a unique solution $y(x, s ; t)$ to $(\mathrm{O})$ for $t \in[0, T]$ and if

$$
\|y\|_{C(D ; T, T)}=\sup _{x \in D ; s, t \in[0, T]}|y(x, s ; t)|
$$

then

$$
\left|y(x, s ; t)-y\left(x, s ; t^{\prime}\right)\right| \leq\left|t-t^{\prime}\right|\left(\left\|v_{, x}\right\|_{C}\|y\|_{C(D ; T, T)}+\|b\|_{C}\right)
$$


and

$\left|y(x, s ; t)-y\left(x^{\prime}, s^{\prime} ; t\right)\right|$

(ii)

$$
\begin{aligned}
\leq & \left(\left|x-x^{\prime}\right|^{q}+\|v\|_{C}^{q}\left|s-s^{\prime}\right|^{q}\right)\left[\|a\|_{C^{q}}+t\left(\|v\|_{C^{1+q, 0}}\|y\|_{C(D ; T, T)}+\|b\|_{C^{q}}\right)\right] \\
& \cdot \exp \left(3 T\left\|v_{, x}\right\|_{C}\right),
\end{aligned}
$$

$$
w(x, t) \equiv y(x, t ; t) \in C^{q, q}(\bar{D}, T) .
$$

Proof. From [2, p. 74], we know that there is a "fundamental matrix" $F_{v}(t)$ for linear system $(\mathrm{O})$ and the solution has form

$$
\begin{aligned}
y(x, s ; t)= & F_{v}(t) F_{v}(0)^{-1} a(X(x, s ; 0)) \\
& +F_{v}(t) \int_{0}^{t} F_{v}^{-1}(\tau) b(X(x, s ; \tau), \tau) d \tau
\end{aligned}
$$

and is a continuous function (for fixed $v$ ) of the parameters $x \in \bar{D}$ and $s \in[0, T]$. Hence $\|y\|_{C(D ; T, T)}$ exists. We use Theorem A and Lemma II-1 to obtain additional smoothness and estimates for $y(x, s ; t)$.

$$
\begin{aligned}
& \left|y(x, s ; t)-y\left(x^{\prime}, s ; t\right)\right| \\
& \leq\left\{\left|y(x, s ; 0)-y\left(x^{\prime}, s ; 0\right)\right|\right. \\
& +t\left(\sup _{t \in[0, T]}\left|v_{, x}(X(x, s ; t), t)-v_{, x}\left(X\left(x^{\prime}, s ; t\right), t\right)\right|\|y\|_{C(D, T ; T)}\right. \\
& \left.\left.+\sup _{t \in[0, T]}\left|b(X(x, s ; t), t)-b\left(X\left(x^{\prime}, s ; t\right), t\right)\right|\right)\right\} \\
& \cdot \exp \left(T \sup _{t \in[0, T]}\left|v_{, x}(X(x, s ; t), t)\right|\right) \\
& \leq\left\{\left|X(x, s ; 0)-X\left(x^{\prime}, s ; 0\right)\right|^{q}\|a\|_{C^{q}}\right. \\
& \left.+\left|X(x, s ; t)-X\left(x^{\prime}, s ; t\right)\right|^{q} t\left(\|v\|_{C^{1+q, 0}}\|y\|_{C(D ; T, T)}+\|b\|_{C^{q, 0}}\right)\right\} \exp \left(T\left\|v_{, x}\right\|_{C}\right) \\
& \leq\left|x-x^{\prime}\right|^{q} \exp \left((q+1) T\left\|v_{, x}\right\|_{C}\right)\left\{\|a\|_{C^{q}}+t\|v\|_{C^{1+q, 0}}\|y\|_{C(D ; T, T)}+\|b\|_{C^{q, 0}}\right\} .
\end{aligned}
$$

That

$$
\left|y(x, s ; t)-y\left(x, s^{\prime} ; t\right)\right|=\left|y(X(x, s ; s), s ; t)-y\left(X\left(x, s^{\prime} ; s\right), s, t\right)\right|
$$

can be shown by reference to the definition of $X$ and $y$; then the Höldercontinuity in $x$ can be used to obtain the result for $s$. Verifying the Lipschitzcontinuity in $t$ is straightforward. These results easily show that $w(x, t) \equiv y(x, t ; t)$ $\in C^{q, q}(\bar{D} ; T)$. 
Proof of Theorem II-1. Let $y(x, t ; t)$ be the solution of $(0)$ of Lemma II-2. Then if $w(x, t)=y(x, t ; t), w(x, 0)=y(x, 0 ; 0)=a(X(x, 0 ; 0))=a(x)$ and

$$
\begin{aligned}
& \left.\frac{d y(x, t ; \tau)}{d \tau}\right|_{\tau=t}=v_{, x}(X(x, t ; t), t) y(x, t ; t)+b(X(x, t ; t), t) \\
& =v_{, x}(x, t) w(x, t)+b(x, t)=(w \cdot \operatorname{grad}) v+b . \\
& \begin{aligned}
\int_{D}(y(x, t+\Delta t ; t)-y(x, t ; t)) f(x) d x \\
=\int_{D}(y(X(x, t+\Delta t ; t), t ; t)-y(X(x, t ; t), t ; t)) f(x) d x \\
=\int y(x, t ; t) f(X(x, t ; t+\Delta t))-f(X(x, t ; t)) d x
\end{aligned}
\end{aligned}
$$

by the measure-preserving and inverse properties of $X(\cdot, s ; t)$ (see Lemma II-1 (ii)). Hence we can multiply by $(\Delta t)^{-1}$ and take the limit to obtain

$$
\lim _{\Delta t \rightarrow 0} \frac{1}{\Delta t} \int_{D}(y(x, t+\Delta t ; t)-y(x, t ; t)) f(x) d x=(w,(v \cdot \operatorname{grad}) f) .
$$

This establishes that $w(x, t)$ is the weak solution required.

III. In this section we apply Schauder's fixed point theorem using the two mappings $F_{1}$ and $F_{2}$ defined in $\S \S I$ and II applied to the set

$$
\begin{aligned}
S=\left\{w \in C^{q, q}(\bar{D} ; T) \mid\|w\|_{C} \leq K_{3} ; k^{q, q}(w)\right. & \leq K_{4} ; \nabla \cdot x=0 \\
w & \left.\left.\in L_{2}(D) \ominus H_{n} \text { (for fixed } t \in[0, T]\right)\right\}
\end{aligned}
$$

defined for suitable constants $K_{3}, K_{4}$ and $T>0$ (see introduction for notation).

Lemma III-1. The set $S$ is a compact convex set in the space $C(\bar{D} ; T)$.

Proof. Convexity is immediate. Compactness in $C(\bar{D} ; T)$ follows from the equicontinuity implied by the requirement $K^{q, q}(w) \leq K_{4}$ and the Arzela-Ascoli theorem and straightforward verification of the closure requirements.

Lemma III-2. Let $C_{1}=C_{1}(q), C_{3}=C_{3}(q), C_{4}=C_{4}(q)$ and

$$
\begin{aligned}
C_{5} & =\left(C_{1}+C_{3} e\right)^{-1} C_{2} e\left(\left\|A_{H}\right\|_{C}+\left\|B_{H}\right\|_{C}\right), \\
K_{3}= & (3 / 2)\|A\|_{C^{4}}+1 / 2, \\
K_{4}= & \left(K_{3}\left(2 e\left(C_{1}+C_{3} e\right)^{q}+1\right)+C_{5}\right)^{1 /(1-q)}-\left(K_{3}+C_{5}\right), \\
T^{1-q} \leq & (1 / 3)\left\{\left(K_{3}+K_{4}\right)\left(C_{1}+C_{3} e+\left(C_{4}\right) / 3\right)\right. \\
& \left.\quad+C_{2} e\left(\left\|A_{H}\right\|_{C}+\left\|B_{H}\right\|_{C}\right)+\|b\|_{C^{q, 0}}\right\}^{-1},
\end{aligned}
$$


and assume $T \leq 1$. Then $w \in F_{2}\left(F_{1}(S)\right)$ implies $\|w\|_{c} \leq K_{3}$ and $K^{q, q}(w) \leq K_{4}$.

Proof. If $w \in S$ and $v=F_{1}(w)$, then Theorem I-1 and the restriction on $T$ gives

$$
\begin{aligned}
\|v\|_{C^{1+q, 0}} & \leq\left(C_{1}+C_{3} e\right)\left(K_{3}+K_{4}\right)+C_{2} e\left(\left\|A_{H}\right\|_{C}+T\left\|B_{H}\right\|_{C}\right) \\
& \leq\left(3 T^{1-q}\right)^{-1} \leq(3 T)^{-1}
\end{aligned}
$$

Let $y(x, s ; t)$ be the solution of system (O) of Lemma II-2 with $v=F_{1}(w)$. Then

$$
\begin{aligned}
y(x, s ; t)-a(X(x, s ; 0)) & \\
& =\int_{0}^{t} v_{, x}(X(x, s ; \tau), \tau) y(x, s ; \tau)+b(X(x, s ; \tau), \tau) d \tau,
\end{aligned}
$$

so

$$
|y(x, s ; t)| \leq\|a\|_{C}+t\left\{\|v\|_{C^{1+r, 0}} \sup _{\tau \in[0, t]}|y(x, s ; \tau)|+\|b\|_{C}\right\} .
$$

Since $y(x, t ; t)=w^{\prime}(x, t) \equiv F_{2}\left(F_{1}(w)\right)$ we get

$$
\begin{aligned}
\left\|w^{\prime}\right\|_{C(\bar{D} ; t)} & \leq\|a\|_{C}+\frac{1}{3}(t / T)\|w\|_{C(\bar{D} ; t)}+t\|b\|_{C} \quad \text { or } \\
\left\|w^{\prime}\right\|_{C(\bar{D} ; T)} & \leq(3 / 2)\|a\|_{C^{+}}+\frac{1}{2}=K_{3} .
\end{aligned}
$$

Similarly

$$
\begin{aligned}
\left|y(x, s ; t)-y\left(x, s ; t^{\prime}\right)\right| & \leq\left|t-t^{\prime}\right|^{q} T^{1-q}\left(\left(K_{3}\right) /\left(3 T^{1-q}\right)+\|b\|_{C}\right) \\
& \leq\left|t-t^{\prime}\right|^{q}\left(K_{3}+1\right) / 3 \leq\left|t-t^{\prime}\right|^{q}\left(K_{4}\right) / 2 .
\end{aligned}
$$

Lemma II-2 gives

$$
\left|y(x, s ; t)-y\left(x^{\prime}, s^{\prime} ; t\right)\right| \leq\left(\left|x-x^{\prime}\right|^{q}+\|v\|_{c^{q}}\left|s-s^{\prime}\right|^{q}\right) e K_{3} .
$$

Hence $\left|w^{\prime}(x, t)-w^{\prime}\left(x^{\prime}, t\right)\right| \leq\left|x-x^{\prime}\right|^{q}\left(e K_{3}\right) \leq\left|x-x^{\prime}\right|^{q} K_{4}$. We also have

$$
\begin{aligned}
& \left|y(x, s ; t)-y\left(x, s^{\prime} ; t\right)\right| \\
& \quad \leq\left|s-s^{\prime}\right|^{q} e K_{3}\left(C_{2} e\left(\left\|A_{H}\right\|_{C}+T\left\|B_{H}\right\|_{C}\right)+\left(K_{3}+K_{4}\right)\left(C_{1}+C_{3} e\right)\right)^{q} .
\end{aligned}
$$

To show that this final inequality yields the desired result, we note that the righthand side has form $\left|s-s^{\prime}\right|{ }^{q} K_{5}\left(K_{6}+K_{4}\right)^{q}$; we require that this be less than $\frac{1}{2} K_{4}$. Since $0<q<1$, the existence of such $K_{4}$ follows from considering the equivalent problem of finding $K_{4}$ such that 


$$
2 K_{5} \leq\left(K_{6}+K_{4}\right)^{1-q}-K_{6}\left(K_{6}+K_{4}\right)^{-q},
$$

which is clearly obtainable for sufficiently large $K_{4}$ and will remain true for larger $K_{4}$. Verification that our estimate is adequate is straightforward.

Lemma III-3. The map $F_{2}\left(F_{1}(\quad)\right.$ is continuous in the $C(\bar{D} ; T)$ topology from $S$ into $C(\bar{D} ; T)$.

Proof. Suppose $w_{i} \in S, i=0,1,2, \ldots$, and $\left\|w_{i}-w_{0}\right\|_{C} \rightarrow 0$ as $i \rightarrow \infty$. Then, using the notation of Theorem I-1, $F_{1}\left(w_{i}\right)=u_{i}+h_{i} \equiv v_{i}$.

First we wish to establish that $\left\{v_{i, x}\right\}$ is an equicontinuous set. To show that $\left\{h_{i, x}\right\}$ is equicontinuous, since $h_{i} \in H_{\tau}$ (finite dimensional) for fixed $t$, it suffices to show that $\left\{h_{i, x}\right\}$ is equicontinuous in $t$; but it is immediate that they are uniformly Lipschitz-continuous from the bounds of Lemma III-2 and the defining ordinary differential equations for $h_{i}$ of Theorem I-1. Equicontinuity of $\left\{u_{i, x}\right\}$ in $x \in \bar{D}$ follows from inequality (i) of Theorem I-1. To show equicontinuity in $t \in[0, T]$, we note that since $w_{i} \in C^{q, q}(\bar{D} ; T), w_{i} \in C^{q-r, q}(\bar{D} ; T)$ for any $r, 0<r<q$, and

$$
\begin{aligned}
& \left|u_{i, x}(x, t)-u_{i, x}(x, s)\right| \\
& \leq C_{1}(q-r)\left(|| w_{i}(\cdot, t)-w_{i}(\cdot, s) \|_{C(\bar{D})}\right. \\
& \left.\quad+\sup _{\substack{x, x^{\prime} \in \bar{D} \\
\left|x-x^{\prime}\right|<1 \\
x \neq x^{\prime}}} \frac{\left|w_{i}(x, t)-w_{i}(x, s)-w_{i}\left(x^{\prime}, t\right)+w_{i}\left(x^{\prime}, s\right)\right|^{(q-r) / q+r / q}}{\left|x-x^{\prime}\right|^{q-r}}\right) \\
& \leq C_{1}(q-r)\left(K_{4}|t-s|^{q}+\frac{\left(2 K_{4}\left|x-x^{\prime}\right|^{q}\right)^{(q-r) / q}\left(2 K_{4}|t-s|^{q}\right)^{r / q}}{\left|x-x^{\prime}\right|^{q-r}}\right) \\
& \leq C_{1}(q-r) 3 K_{4}|t-s|^{r} .
\end{aligned}
$$

We wish to show that $\left\|v_{i}-v_{0}\right\|_{C^{1,0}} \rightarrow 0$ as $i \rightarrow \infty$. Suppose not. Then, using the equicontinuity just established and the Arzela-Ascoli theorem, there is a subsequence $\left\{v_{j}\right\}$ and $v^{\prime} \in C^{1,0}(\bar{D} ; T)$ and $\varepsilon>0$ such that $\left\|v_{j}-v_{0}\right\|_{C^{1,0}} \geq \varepsilon$ and $\left\|v_{j}-v^{\prime}\right\|_{C^{1,0}} \rightarrow 0$ as $j \rightarrow \infty$. However, the criteria for the existence of a unique $v_{0}$ satisfying the constraints of Theorem I-1 are maintained under $C^{1,0}(\bar{D} ; T)$ convergence (defining $h^{\prime} \equiv \sum\left(v^{\prime}, h^{i}\right) h^{i}$; etc.), hence $v^{\prime}=v_{0}$ and we have a contradiction.

Let $X_{i}(x, s ; t)$ be the streamline associated with $v_{i}$ and

$$
w_{i}^{\prime}=F_{2}\left(F_{1}\left(w_{i}\right) \equiv y_{i}(x, t ; t)\right.
$$

where $y_{i}(x, s ; t)$ is the solution to system $(\mathrm{O})$ defined by $v_{i}$. Then Theorem A gives

$$
\left|X_{i}(x, s ; t)-X_{0}(x, s ; t)\right| \leq|t-s|\left\|v_{i}-v_{0}\right\|_{c} e^{1 / 3}
$$

and, using this, 


$$
\begin{aligned}
& \left|y_{i}(x, s ; t)-y_{0}(x, s ; t)\right| \\
& \leq\left\{\left|a\left(X_{i}(x, s ; 0)\right)-a\left(X_{0}(x, s ; 0)\right)\right|\right. \\
& +t \sup _{\tau \in[0, t]}\left(K_{3}\left|v_{i, x}\left(X_{i}(x, s ; \tau), \tau\right)-v_{0, x}\left(X_{0}(x, s ; \tau), \tau\right)\right|\right. \\
& \left.\left.+\left|b\left(X_{i}(x, s ; \tau), \tau\right)-b\left(X_{0}(x, s ; \tau), \tau\right)\right|\right)\right\} \exp \left(T\left\|v_{0, x}\right\|_{C}\right) \\
& \leq e\left\{\left(\|a\|_{C^{q}}+T K^{q, 0}(b)\right)\left(T\left\|v_{i}-v_{0}\right\|_{C} e^{1 / 3}\right)^{q}\right. \\
& +T K_{3}\left(\operatorname { s u p } _ { t \in [ 0 , T ] } \left(\left|v_{i, x}\left(X_{i}(x, s ; t), t\right)-v_{i, x}\left(X_{0}(x, s ; t), t\right)\right|\right.\right. \\
& \left.\left.\left.+\left|v_{i, x}\left(X_{0}(x, s ; t), t\right)-v_{0, x}\left(X_{0}(x, s ; t), t\right)\right|\right)\right)\right\} \\
& \leq e\left\{\|a\|_{C^{q}}+T K^{q, 0}(b)+\left(K_{3} / 3\right)\left(T\left\|v_{i}-v_{0}\right\|_{C} e^{1 / 3}\right)^{q}+T K_{3}\left\|v_{i}-v_{0}\right\|_{C^{1,0}}\right\}
\end{aligned}
$$

from the restriction on $T$ (see Lemma III-2). Since $\left\|v_{i}-v_{0}\right\|_{c^{1,0}} \rightarrow 0$ as $i \rightarrow \infty$, this establishes the continuity.

Lemma III-4. If $w \in F_{2}\left(F_{1}(S)\right)$, then $\nabla \cdot w=0$ (in the generalized sense).

Proof. If we assume $v \in C^{3,0}(\bar{D} ; T), b \in C^{2,0}(\bar{D} ; T)$ and $a \in C^{2}(\bar{D})$ then, by standard results concerning differentiable dependence on parameters of ordinary differential equations [2, p. 31] applied to both the streamlines and the system (O), $y(x, t ; t) \equiv w(x, t) \in C^{2,1}(\bar{D} ; T)$ and so the weak solution of Theorem II-1 of (E) (a), (b) is classical. Hence we can take the divergence of system (E) (a), (b) to obtain $(\partial / \partial t)(\nabla \cdot w)+v \cdot \operatorname{grad}(\nabla \cdot w)=0$ and $\nabla \cdot w(x, 0)=\nabla \cdot(\nabla \times A)=0$ since several terms cancel and $\nabla \cdot v=0=\nabla: b$. Now, to show that $\nabla \cdot w(x, t)$ $=0$ it is sufficient to show that $\nabla \cdot w(X(x, s ; t), t)=0$ since $X(x, s ; t)$ is a $1-1$ map of $\bar{D}$ onto $\bar{D}$ for fixed $s$ and $t$.

$$
0=\nabla \cdot w(X(x, s ; 0), 0)
$$

and

$$
\begin{aligned}
\frac{d}{d t} \nabla \cdot w(X(x, s ; t), t) & =\frac{\partial}{\partial t}(\nabla \cdot w(X(x, s ; t), t))+\left(\frac{\partial}{\partial x_{i}}(\nabla \cdot w)\right) \cdot v_{i} \\
& =\frac{\partial}{\partial t}(\nabla \cdot w)+v \cdot \operatorname{grad}(\nabla \cdot w)=0
\end{aligned}
$$

was just established. Hence $\nabla \cdot w=0$.

For our purpose it is sufficient to show that $(w, \nabla g)=0$ for any smooth scalarvalued function $g$ with support in some compact $D^{\prime} \subset D$. Using Friedrich's mollifier we can construct sequences $\left\{a^{i}\right\},\left\{b^{i}\right\}$ and $\left\{V^{i}\right\}$ of the required smooth- 
ness that preserve the relations $\nabla \cdot a^{i}=0 ; \nabla \cdot b^{i}=0$ and $\nabla \cdot v^{i}=0$ and converge uniformly together with the necessary derivatives to $a, b$ and $v$ respectively on any compact set $D^{\prime \prime} \subset D$. Although the associated streamlines $X^{i}(x, s ; t)$ may lead out of the original compact set $D^{\prime}$, the continuity arguments of Lemma III-3 and the result that $X(x, s ; t)$ takes $b D$ into $b D$ of Lemma II-1(ii) show that by taking $v^{i}$ sufficiently close to $v$ in a chosen set $D^{\prime \prime}$ compactly contained in $D$ and containing $D^{\prime}$ in its interior, streamlines starting in $D^{\prime}$ will stay in $D^{\prime \prime}$ for $s$ and $t \in[0, T]$. Hence we can define solutions $w^{i}$ of (E)(a), (b) using $a^{i}, b^{i}$ and $v^{i}$ and the previous reasoning will yield $\nabla \cdot w^{i}=0$ in $D^{\prime}$. The continuity arguments of Lemma III-3 show the dependence of $w^{i}$ on $v^{i}$ and the representation of solutions $y^{i}(x, s ; t)$ to (O) with $v^{i}$ of Lemma II-2 show the dependence on $a^{i}$ and $b^{i}$; together these allow us to assert that $w^{i}$ approaches $w$ uniformly in $D^{\prime} \times[0, T]$ and hence $(w, \nabla g)=0$.

Lemma III-5. $w \in F_{1}\left(F_{2}(S)\right)$ implies that $w \in L_{2}(D) \ominus H_{n}$ (for fixed $t \in[0$, T]).

Proof. First, note that if $h \in H_{n}$ and hence $h=g(x) n$ on $b D$ where $g(x)$ is a scalar $(\nabla \times F, h)=(F, \nabla \times h)=0$ for any $F \in C^{\prime}(\bar{D})$.

Now, if $w$ is the weak solution of (E)(a), (b) of Theorem II-1

$$
(d / d t)(w, h)=(\nabla \times B, h)+(w,(v \cdot \operatorname{grad}) h)+((w \cdot \operatorname{grad}) v, h)
$$

and

$$
\begin{aligned}
(w(0), h) & =(\nabla \times A, h)=0, \\
((w \cdot \operatorname{grad}) v, h) & =(w, \nabla(v \cdot h))-(v,(w \cdot \operatorname{grad}) h) .
\end{aligned}
$$

But, on $b D, v \cdot h=g(x) v \cdot n=0$. Hence, using Lemma III-4 and the limit argument employed in Theorem $\mathrm{I}-1,(w, \nabla(v \cdot h))=0$. Then, since $(\nabla \times B, h)=0$

$$
\frac{d}{d t}(w, h)=\int_{D}\left(\sum_{i, j} w_{i} v_{j} \frac{\partial h_{i}}{\partial x_{j}}-v_{j} w_{i} \frac{\partial h_{j}}{\partial x_{i}}\right) d x=0
$$

since $\nabla \times h=0$ implies $\partial h_{i} / \partial x_{j}=\partial h_{j} / \partial x_{i}$. Hence $(w, h)=0$ for all $t \in[0, T]$.

Theorem III-1. If $A \in C^{1+q}(\bar{D}), \nabla \cdot A=0, A \cdot n=0$ on $b D, B \in C^{1+q, 0}(\bar{D} ; T)$, then there exists a unique $v \in C^{1+q, r}(\bar{D} ; T), 0<r<q<1$, such that $\partial v / \partial t$ exists in $C^{q, 0}(\bar{D} ; T)$ and $P$ exists with $\nabla P \in C^{q, 0}(\bar{D} ; T)$, unique up to a function of that may be added to $P$ such that

$$
\begin{gathered}
\partial v / \partial t+(v \cdot \operatorname{grad}) v=-\operatorname{grad} P+B \\
\nabla \cdot v=0, \quad v \cdot n=0 \quad \text { on } b D, \text { and } v(x, 0)=A(x)
\end{gathered}
$$

for $t \in[0, T]$ with $T$ satisfying the constraints of Lemma III-2. 
Proof. With $A$ and $B$ construct the mappings $F_{1}(\quad)$ and $F_{2}(\quad)$ of Theorems I-1 and II-1. The previous lemmas establish that the requirements for applying Schauder's fixed point theorem to the mapping $F_{2}\left(F_{1}(\cdot)\right)$ from $S$ (defined in the beginning of this section) into itself are met and hence there is a fixed point $w \in S$. Let $v=F_{1}(w)$; then $v$ and $\nabla \times v=w$ give the weak solution to system (E) of Theorem II-1. To establish the differentiability in $t$ of $v$, we note that for any $f \in C^{1+q}(\bar{D})$ we can use the representation result of Morrey [6] mentioned in Theorem I-1 to obtain $f=\nabla \times\left(\nabla \times p_{f}^{-}\right)-\nabla\left(\nabla \cdot p_{f}^{-}\right)+h_{f}^{-}$. Then

$$
\begin{aligned}
(v, f) & =\left(v, \nabla \times\left(\nabla \times p_{f}^{-}\right)\right)-\left(v, \nabla\left(\nabla \cdot p_{f}^{-}\right)\right)+\left(v, h_{f}^{-}\right) \\
& =\left(\nabla \times v, \nabla \times p_{f}^{-}\right)+\left(v, h_{f}^{-}\right)=\left(w, \nabla \times p_{f}^{-}\right)+\left(v, h_{f}^{-}\right) .
\end{aligned}
$$

The $t$-derivatives of the two terms on the right-hand side exist by Theorems I-1 and II-1 and we get, letting $\nabla \times p_{f}^{-}=Q$,

$$
\begin{aligned}
(d / d t)(v, f)= & (d / d t)(w, Q)+\left(-(v \cdot \operatorname{grad}) v+B, h_{f}^{-}\right) \\
(d / d t)(w, Q)= & (\nabla \times v,(v \cdot \operatorname{grad}) Q)+(((\nabla \times v) \cdot \operatorname{grad}) v, Q)+(\nabla \times B, Q) \\
= & (\nabla \times v,(v \cdot \operatorname{grad}) Q)-(v,((\nabla \times v) \cdot \operatorname{grad}) Q) \\
& +(\nabla \times v, \nabla(v \cdot Q))+(B, \nabla \times Q) \\
= & (-(v \cdot \operatorname{grad}) v, \nabla \times Q)+(\nabla(v \cdot v), \nabla \times Q)+(B, \nabla \times Q) \\
= & (-(v \cdot \operatorname{grad}) v+B, \nabla \times Q)
\end{aligned}
$$

since $v \cdot Q=0$ on $b D$ and $\nabla \times Q \cdot n=0$ on $b D$. Now $R \equiv-(v \cdot \operatorname{grad}) v+B$ $\in C^{q, 0}(\bar{D} ; T$.) We can use the previous representation theorem to obtain $R$ $=\nabla \times\left(\nabla \times p_{R}^{-}\right)-\nabla\left(\nabla \cdot p_{R}^{-}\right)+h_{R}^{-}$. Then

$$
(d / d t)(v, f)=\left(R, \nabla \times\left(\nabla \times p_{f}^{-}\right)+h_{f}^{-}\right)=\left(\nabla \times\left(\nabla \times \overline{p_{R}^{-}}\right)+h_{R}^{-}, f\right) .
$$

Hence $d v / d t$ exists in $C^{q, 0}(\bar{D}, T) . d v / d t=\nabla \times\left(\nabla \times p_{R}^{-}\right)+h_{R}^{-}$and furthermore $d v / d t+(v \cdot \operatorname{grad}) v-B=\nabla\left(\nabla \cdot p_{R}^{-}\right)$. We let $P=-\nabla \cdot p_{R}^{-}$and our solution is obtained. Also,

$$
\begin{aligned}
(v(0), f) & =\left(w(0), \nabla \times \overline{p_{f}^{-}}\right)+\left(A, h_{f}^{-}\right) \\
& =\left(\nabla \times A, \nabla \times p_{f}^{-}\right)+\left(A, h_{f}^{-}\right) \\
& =\left(A, \nabla \times\left(\nabla \times \overline{p_{f}^{-}}\right)-\nabla\left(\nabla \cdot p_{f}^{-}\right)+h_{f}^{-}\right) \\
& =(A, f)
\end{aligned}
$$

since $\left(A, \nabla\left(\nabla \cdot p_{f}^{-}\right)\right)=0(\nabla \cdot A=0$ and $A \cdot n=0$ on $b D)$. This establishes that the initial value is achieved. To establish uniqueness: suppose $v_{i}, P_{i}$ are solutions, $i=1,2$. Then, letting $\bar{v}=v_{1}-v_{2}, \bar{P}=P_{1}-P_{2}$, 


$$
d \bar{v} / d t+(\bar{v} \cdot \operatorname{grad}) v_{1}+\left(v_{2} \cdot \operatorname{grad}\right) \bar{v}=-\nabla \bar{P} .
$$

Taking the $L_{2}$ inner-product with $\bar{v}$ and noting that $\left(\left(v_{2} \cdot \operatorname{grad}\right) \bar{\nu}, \bar{v}\right)=0=(-\nabla \bar{P}$, $\bar{v}$ ), we get

$$
(d / d t) \frac{1}{2}\|\bar{\nu}\|_{L_{2}}^{2}=\left(-(\bar{v} \cdot \operatorname{grad}) v_{1}, \bar{v}\right) \leq K\left\|v_{1, x}\right\|_{C}\|\bar{v}\|^{2}
$$

with $\bar{v}(0)=0$. Such a relationship can hold only if $\bar{v}=0$. The qualified uniqueness of $P$ follows from the uniqueness of $v$.

\section{BIBLIOGRAPHY}

1. G. Birkhoff and G. C. Rota, Ordinary differential equations. Introduction to higher mathematics, Ginn, Boston, Mass., 1962. MR 25 \# 2253.

2. E. A. Coddington and N. Levinson, Theory of ordinary differential equations, McGraw-Hill, New York, 1955. MR 16, 1022.

3. D. G. Ebin and J. E. Marsden, Groups of diffeomorphisms and the motion of an incompressible fluid, Ann. of Math. (2) 92 (1970), 102-163. MR 42 \#6865.

4. T. Kato, On classical solutions of the two-dimensional non-stationary Euler equation, Arch. Rational Mech. Anal. 25 (1967), 188-200. MR 35 \# 1939.

5. L. Lichtenstein, Grundlagen der Hydromechanik, Springer-Verlag, Berlin, 1929.

6. C. B. Morrey, Jr., Multiple integrals in the calculus of variations, Die Grundlehren der math. Wissenschaften, Band 130, Springer-Verlag, New York, 1966. MR 34 \#2380.

7. H. S. G. Swann, The convergence with vanishing viscosity of nonstationary Navier-Stokes flow to ideal flow in $R_{3}$, Trans. Amer. Math. Soc. 157 (1971), 373-397. MR 43 \# 3662.

Department of Mathematics, California State University at San Jose, San Jose, CaliforNIA 95192 